\title{
Finding Top-k Similar Pairs of Objects Annotated with Terms from an Ontology
}

\author{
Arnab Bhattacharya \\ arnabb@iitk.ac.in \\ Dept. of Computer Science and Engineering, \\ Indian Institute of Technology, Kanpur \\ Kanpur, UP 208016, India.
}

\author{
Abhishek Bhowmick \\ bhowmick@iitk.ac.in \\ Dept. of Computer Science and Engineering, \\ Indian Institute of Technology, Kanpur \\ Kanpur, UP 208016, India.
}

\author{
Ambuj K. Singh \\ ambuj@cs.ucsb.edu \\ Dept. of Computer Science, \\ University of California, Santa Barbara \\ Santa Barbara, CA 93106, USA.
}

\begin{abstract}
With the growing focus on semantic searches and interpretations, an increasing number of standardized vocabularies and ontologies are being designed and used to describe data. We investigate the querying of objects described by a tree-structured ontology. Specifically, we consider the case of finding the top- $k$ best pairs of objects that have been annotated with terms from such an ontology when the object descriptions are available only at runtime. We consider three distance measures. The first one defines the object distance as the minimum pairwise distance between the sets of terms describing them, and the second one defines the distance as the average pairwise term distance. The third and most useful distance measure - earth mover's distance-finds the best way of matching the terms and computes the distance corresponding to this best matching. We develop lower bounds that can be aggregated progressively and utilize them to speed up the search for top- $k$ object pairs when the earth mover's distance is used. For the minimum pairwise distance, we devise an algorithm that runs in $O(D+T k \log k)$ time, where $D$ is the total information size and $T$ is the total number of terms in the ontology. We also develop a novel best-first search strategy for the average pairwise distance that utilizes lower bounds generated in an ordered manner. Experiments on real and synthetic datasets demonstrate the practicality and scalability of our algorithms.
\end{abstract}

\section{Introduction}

We are witnessing an unprecedented growth in annotated information. This growth has been motivated by a need to share information and, more recently, by a need to search and analyze objects based on their structure and seman- tics. Annotated objects occur in multiple application domains including language (http://wordnet.princeton.edu/), biology (http://www.geneontology.org), medical documents (http://www.nlm.nih.gov/mesh/), web content (http://www.semanticweb.org/), etc. In all these cases, annotations are derived from a structured vocabulary or ontology. An ontology uses a number of different relationships (e.g., is-a, is-part-of) to organize concepts or hierarchies.

This paper investigates the analysis of large sets of objects that have been annotated with terms from a common ontology. The basic problem we consider is as follows: Given two sets of objects annotated with terms from a common ontology, how to find the top- $k$ pairs of objects among the two sets that are most similar.

The above problem statement requires us to formalize the notion of distance between two terms in a given ontology and then to extend this notion to distance between two annotated objects. The distance between two terms can be measured by the shortest path distance on the ontology.

There are a number of definitions for distance (or conversely, similarity) between objects. Two obvious definitions are based on the minimum pairwise distance and the average pairwise distance between the annotations. The third one is the earth mover's distance [12] that takes into account the relative positions of the terms that describes the objects. We investigate querying based on these three distance measures.

In this paper, we consider that the object descriptions are submitted in an online fashion, i.e., they are available only at run-time. As such, no pre-processing or index construction or any other offline processing can be used, and all the computation costs are paid at run-time. Even if the distance function used is a metric, the online nature of the problem renders the use of index structures like the M-tree [3] infeasible due to their high index construction 


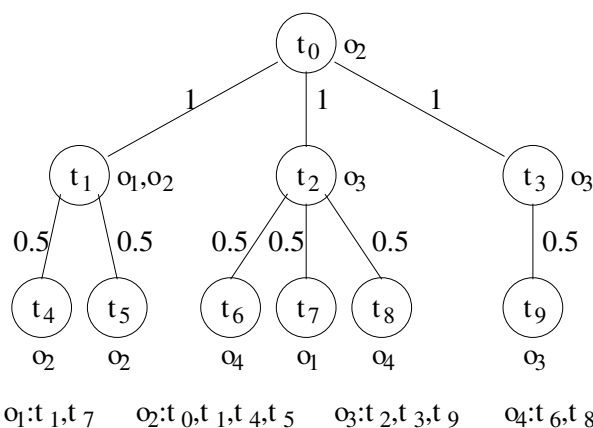

Figure 1: Example of an ontology tree with objects.

times. In a way, this problem is reminiscent of the computation of spatial joins on objects embedded in the Euclidean space: the spatial datasets are delivered online and we need to compute the best spatial matches [16]. Only that, in the case of ontologies, the primitive distance is not Euclidean, but computed on a tree.

The problem we consider here can be extended easily to the case when objects are annotated with multiple independent ontologies. We can compute the per-ontology distance and combine them using an aggregate ranking technique such as the threshold algorithm [7]. The problem of finding objects similar to a given query object (i.e., the $k$-NN problem) reduces to the special case of a join of the database with a singleton set, the query object. Similarly, range queries can be solved by choosing only those pairs having a distance less than the query range. While these and other kinds of queries can also be considered in our setting, the problem of top- $k$ joins exposes the computational and data management complexities of this domain well, making it the right problem to consider.

Formally, our problem can be stated as:

Problem 1. Given a set of objects each of which is defined by a set of terms from an ontology and a distance function $d\left(O_{i}, O_{j}\right)$ between two objects $O_{i}$ and $O_{j}$, find $k$ pairs of objects $P$ such that for any $\left(O_{i}, O_{j}\right) \in P$ and $\left(O_{g}, O_{h}\right) \notin$ $P, d\left(O_{i}, O_{j}\right) \leq d\left(O_{g}, O_{h}\right)$

Figure 1 illustrates a particular instance of the problem. The ontology tree consists of 10 terms. There are 4 objects that are described by these terms. The object descriptions are given by $O_{1}=\left\{t_{1}, t_{7}\right\}, O_{2}=\left\{t_{0}, t_{1}, t_{4}, t_{5}\right\}$, $O_{3}=\left\{t_{2}, t_{3}, t_{9}\right\}, O_{4}=\left\{t_{6}, t_{8}\right\}$. An inverted index, i.e., mapping a term to set of objects can be maintained on the ontology itself (as shown in the figure). Thus, each node in the tree statically maintains a list $L$ of the objects that are described using the term corresponding to the node. For example, the list of objects for $t_{0}$ is $\left(\mathrm{O}_{2}\right)$. We will use term and node interchangeably to denote the node in which the term resides.
The edge weights on the tree decrease exponentially as the level increases. Concepts closer to the root of the ontology are less similar than concepts that share some common ancestors. For example, broader concepts such as "sports" and "politics" should be more dissimilar than relatively narrower concepts such as "football" and "cricket". The exponentially decreasing edge weights capture this notion. We highlight the fact that the exponential edge weighting function is an example, and not a necessity for the algorithms to work. They produce correct answers for all edge weights.

We denote the number of objects by $N$, the number of terms by $T$, the total information size (i.e., the total number of describing terms for all the objects) by $D$, and the number of object pairs queried by $k$. In Figure $11, N=$ $4, T=10$, and $D=11$.

Our contributions in this paper are as follows:

1. First, we propose the problem of finding top- $k$ most similar object pairs that are annotated with terms in a hierarchy in an online fashion. The terms may define concepts in an ontology and objects may be described using the concepts.

2. Then, we define and motivate three different distance functions (equivalently, similarity measures) that can be used to describe the similarity between a pair of objects. The minimum pairwise distance is useful for searching objects sharing a similar term (concept). The average pairwise distance can be used to query objects that are described using multiple attributes. The earth mover's distance (EMD) finds the best way of matching the terms from two objects and finds the distance corresponding to this best matching.

3. Finally, we develop efficient algorithms to solve the problem using the above distances. We use lower bounds based on $L_{1}$ on reduced number of terms to speed up the computation of EMD. The $L_{1}$ distance, in turn, is computed progressively using a modified version of the threshold algorithm. For the minimum pairwise distance, we show that the top- $k$ query runs in $O(T k \log k)$ time, where $T$ is the size of the ontology. For the average pairwise distance, we devise an efficient best-first search algorithm that avoids distance computations by generating lower bounds in an ordered manner. Experimental evaluations demonstrate the scalability and practicality of our algorithms.

The rest of the paper is organized as follows. Section 2 describes the related work. Section 3 defines the term 
distance and the different object distances. Sections 4, 5 and 6 present the different algorithms for finding the top$k$ pairs of objects using those distances. Experimental results are discussed in Section 7 . Section 8 concludes the paper.

\section{Related Work}

Heterogeneous and high-throughput data is becoming commonplace in the sciences and there is consensus that integration of this information is needed for new breakthroughs. In all these cases, annotations are derived from a structured vocabulary or ontology. The Semantic Web (http://www.semanticweb.org/) has defined a specific language, OWL (http://www.w3.org/2004/OWL/), for describing ontologies. In biology, genes are described using Gene Ontology (GO) (http://www.geneontology.org/) that annotates genes and gene products by three kinds of terms reflecting molecular functions, biological processes, and cellular components. Millions of abstracts in Pubmed (http://www.pubmed.gov/) are indexed using MESH terms (http://www.nlm.nih.gov/mesh/). WordNet (http://wordnet.princeton.edu/) is a lexical database that groups English words into cognitive synonyms (or synsets). Hundreds of other ontologies have been proposed over diverse application domains such as plant structures (http://www.plantontology.org/), description and publication of digital documents (http://www.dublincore.org/), and earth and the environment (http://sweet.jpl.nasa.gov/ontology/). A good compendium of different ontologies is maintained at http://www.ontologyonline.org/.

A given ontology uses a number of different relationships to organize concepts or hierarchical relationships. Of these, "is-a" and "is-part-of" relationships are the most prevalent. The former describes a subsumption relationship while the latter represents how objects combine together to form composite objects. Both of these lead to hierarchical structures in which the proximity between terms (concepts) grows as we descend down the hierarchy.

There have been numerous works on gene ontology ranging from gene function prediction using information theory [18] to defining similarity among genes using the full graph structure of GO [5]. In [8], a comparison of three different gene similarity measures were presented. Probabilistic approaches have also been used [13]. Biologists have used average and minimum pairwise distances between genes based on GO for comparing co-evolutionary rates of yeast genes [14] and for coclustering with gene expression data [2] respectively.
There are a number of similar efforts in the area of information retrieval where the similarity between documents is measured by considering the overlap of terms. The term-frequency inverse-document-frequency (tf-idf) measures consider the frequency of terms in documents [17]. Work on text matching showed that hierarchybased measures using tf-idf outperform lexical similarity measures [15]. Latent Semantic Indexing (LSI) [6] transforms documents into an Euclidean space indexed by latent semantic dimensions. EMD has been shown to be better than other measures in finding document similarities using the WordNet ontology [19].

Embedding an ontology into an Euclidean space [9] and processing queries in the embedding space is another alternative. However, an object description will then span multiple points leading to possibly large MBRs. Further, the approach may suffer from high distortion of the embedding.

In this paper, we tackle the computational challenge of answering queries efficiently using distances defined on hierarchical structures like ontologies.

\section{Distance Definitions}

\subsection{Distance between Terms}

The distance $d\left(t_{i}, t_{j}\right)$ between two terms $t_{i}$ and $t_{j}$ is defined as the length of the path between them on the ontology tree. Since there is only one path between two terms in a tree, from the properties of the shortest path, this distance is a metric [4].

An interesting and important point to note is that when the term distances decrease exponentially at each level, the distance between two terms at the leaves of two subtrees can be approximated by the distance between the roots of the subtrees. For example, in Figure 1 where the edge distances are halved at each level, the distance between $t_{4}$ and $t_{6}(=3)$ can be approximated by that between $t_{1}$ and $t_{2}(=2)$. Using this term distance, we next define different distance measures between the objects. Once more, we emphasize the fact that our algorithms are general enough to work correctly with all edge weights, and not just the exponential function.

We next define the three distance measures $-d_{m i n}$, $d_{\text {avg }}$ and $d_{e m d}$ - between two objects 1

\footnotetext{
${ }^{1}$ We use the terms $d_{\min }$ and MinDist, $d_{a v g}$ and AvgDist, $d_{e m d}$ and EMD interchangeably in the paper.
} 


\begin{tabular}{|c|cccc|}
\hline Min & $O_{1}$ & $O_{2}$ & $O_{3}$ & $O_{4}$ \\
\hline$O_{1}$ & 0.0 & 0.0 & 0.5 & 1.0 \\
$O_{2}$ & & 0.0 & 1.0 & 1.5 \\
$O_{3}$ & & & 0.0 & 0.5 \\
$O_{4}$ & & & & 0.0 \\
\hline
\end{tabular}

Table 1: Minimum pairwise distances for the example in Figure 1

\begin{tabular}{|c|cccc|}
\hline Avg & $O_{1}$ & $O_{2}$ & $O_{3}$ & $O_{4}$ \\
\hline$O_{1}$ & 1.25 & 1.50 & 2.08 & 1.75 \\
$O_{2}$ & & 0.75 & 2.17 & 2.50 \\
$O_{3}$ & & & 1.11 & 2.00 \\
$O_{4}$ & & & & 0.50 \\
\hline
\end{tabular}

Table 2: Average pairwise distances for the example in Figure 1

\subsection{Minimum Pairwise Distance}

Definition 1 (Minimum Pairwise Distance). The minimum pairwise distance between two objects $O_{i}$ and $O_{j}$, denoted by MinDist, is defined as:

$$
d_{\text {min }}\left(O_{i}, O_{j}\right)=\min _{t_{i} \in O_{i}, t_{j} \in O_{j}}\left\{d\left(t_{i}, t_{j}\right)\right\}
$$

This distance is useful when searching objects that have similar terms. For example, even though a single biological document may contain references to different terms like photoreceptor cells and ganglion cells, it is useful to be able to retrieve it when another document that describes photoreceptor cells is queried.

This distance is of particular use in keyword searching, where the query document consists of only the single keyword, and all documents having that keyword will be returned with a distance of 0 . MinDist, in general, extends this idea by finding additional documents that contain terms most similar to the queried keyword.

The MinDist measure is heavily used in hierarchical bottom-up clustering methods where in each step, two clusters with the minimum pairwise distance are merged. It has also been successfully used for finding the distance between two genes, where a gene is annotated with a set of terms from GO [2].

Table 1 shows the MinDist measures among the objects in Figure 11 MinDist is not a metric distance as it does not maintain the triangular inequality. For example, $d_{\text {min }}\left(O_{1}, O_{4}\right)+d_{\text {min }}\left(O_{1}, O_{2}\right)=1.0+0.0<1.5=$ $d_{\min }\left(\mathrm{O}_{2}, \mathrm{O}_{4}\right)$.

\subsection{Average Pairwise Distance}

Definition 2 (Average Pairwise Distance). The average pairwise distance between two objects $O_{i}$ and $O_{j}$, de-

\begin{tabular}{|c|cccc|}
\hline EMD & $O_{1}$ & $O_{2}$ & $O_{3}$ & $O_{4}$ \\
\hline$O_{1}$ & - & 1.25 & 1.75 & 1.75 \\
$O_{2}$ & & - & 2.17 & 2.50 \\
$O_{3}$ & & & - & 2.00 \\
$O_{4}$ & & & & - \\
\hline
\end{tabular}

Table 3: EMDs for the example in Figure1.

noted by AvgDist, is defined as:

$$
d_{\text {avg }}\left(O_{i}, O_{j}\right)=\frac{1}{\left|O_{i}\right| \cdot\left|O_{j}\right|} \sum_{t_{i} \in O_{i}, t_{j} \in O_{j}} d\left(t_{i}, t_{j}\right)
$$

where $\left|O_{i}\right|$ and $\left|O_{j}\right|$ denote the number of terms describing $O_{i}$ and $O_{j}$ respectively.

The AvgDist is useful in cases where the objects are not precisely defined. For example, it has been successfully used for gene function prediction using GO terms for yeast genes [14] as well as in the domain of web services [11]. The MinDist measure fails in such cases.

Table 2 shows the AvgDist measures among the objects in Figure 1 AvgDist is not a metric, as it fails to satisfy the identity property, i.e., $d_{\text {avg }}(x, x)$ can be greater than 0 (e.g., $d_{\text {avg }}\left(O_{1}, O_{1}\right)=1.25$ ). However, since it follows symmetry and triangular inequality 2 , it can be considered as a pseudo-metric distance.

\subsection{Earth Mover's Distance}

Apart from the property of not being a true metric, AvgDist also suffers from the fact that each term in one object is matched with every other term in the other object. For example, consider two documents with the terms $\{$ war, sports $\}$ and $\{$ war, football $\}$. Even though it is obvious that the distance between these two documents should be small, the average distance unnecessarily compares "war" in the first document with "football" in the other. The earth mover's distance (EMD) [12] rectifies this shortcoming by comparing only the like terms through finding the best matching between the terms of the two documents. For this example, EMD will match "war" with "war" and "sports" with "football" and aggregate these distances only. EMD has been shown to be better than other distances in finding similar documents using the WordNet ontology [19].

Formally, each object is considered to be composed of "mass" at the specific spatial locations (corresponding to the terms that describe the object) in the ontology. The total mass of each object is 1 ; consequently, the mass at

\footnotetext{
${ }^{2}$ See Appendix for the proof.
} 


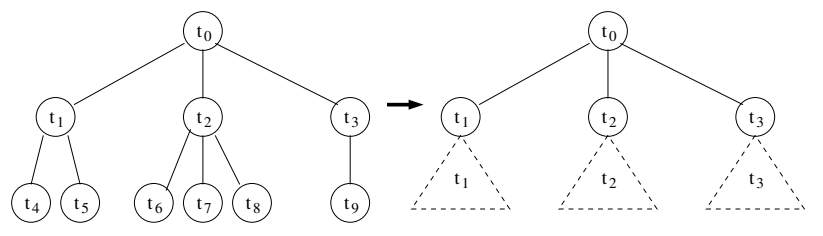

Figure 2: Reduction of terms.

each term location is inverse of the number of terms describing the object. For example, $O_{1}$ in Figure 1 will have mass $\frac{1}{2}$ corresponding to terms $t_{1}$ and $t_{7}$.

The EMD between two objects $A$ and $B$ is the minimum work required to transform $A$ to $B$, where one unit of work is equal to moving one unit of mass through one unit of distance in the ontology. Finding the best "flows" (i.e., how much mass needs to be moved from one term in $A$ to another term in $B$ ) is a linear programming (LP) problem.

Definition 3 (Earth Mover's Distance). The earth mover's distance between two objects $O_{i}$ and $O_{j}$, denoted by EMD, is defined as:

$$
\begin{gathered}
d_{e m d}\left(O_{i}, O_{j}\right)=\min _{f} \sum_{t_{p} \in O_{i}} \sum_{t_{q} \in O_{j}} c_{p q} f_{p q} \\
\text { s.t., each } f_{p q} \geq 0, \\
\forall_{t_{p} \in O_{i}}, \sum_{t_{q} \in O_{j}} f_{p q}=O_{i_{p}}, \text { and } \forall_{t_{q} \in O_{j}}, \sum_{t_{p} \in O_{i}} f_{p q}=O_{j_{q}}
\end{gathered}
$$

where $c_{p q}$ is the ground distance between the terms $t_{p}$ and $t_{q}$ as per the ontology tree and $O_{i_{p}}$ is the mass of $t_{p}$ in $O_{i}$.

EMD is a metric when the ground distance is a metric (proof in [12]). Table 3 shows the EMDs among the objects in Figure 1 .

\subsection{Comparison of the Distance Measures}

To compare the usefulness of the three distance measures, we performed the following experiment. We used WordNet (http://wordnet.princeton.edu/) as the ontology and the "bag-of-words" dataset from the UCI repository (http://archive.ics.uci.edu/ml/datasets/Bag+of+Words) as the set of objects. We chose the first 59 documents from the categories enron and kos of the bag-of-words dataset. Each document was described using nouns from the WordNet ontology, and the ontology was converted into a tree. The top-50 pairs were obtained using all the three distances. For EMD, on an average, there were 45 pairs where both the objects were from the same category. Also, all 10 out of the top-10 pairs were of this nature.

\begin{tabular}{|c|c|c|}
\hline Object & Before & After \\
& $\left\{t_{0}, t_{1}, t_{2}, t_{3}, t_{4}, t_{5}, t_{6}, t_{7}, t_{8}, t_{9}\right\}$ & $\left\{t_{0}, t_{1}, t_{2}, t_{3}\right\}$ \\
\hline$O_{1}$ & $\left\{0, \frac{1}{2}, 0,0,0,0,0, \frac{1}{2}, 0,0\right\}$ & $\left\{0, \frac{1}{2}, \frac{1}{2}, 0\right\}$ \\
$O_{2}$ & $\left\{\frac{1}{4}, \frac{1}{4}, 0,0, \frac{1}{4}, \frac{1}{4}, 0,0,0,0\right\}$ & $\left\{\frac{1}{4}, \frac{3}{4}, 0,0\right\}$ \\
$O_{3}$ & $\left\{0,0, \frac{1}{3}, \frac{1}{3}, 0,0,0,0,0, \frac{1}{3}\right\}$ & $\left\{0,0, \frac{1}{3}, \frac{2}{3}\right\}$ \\
$O_{4}$ & $\left\{0,0,0,0,0,0, \frac{1}{2}, 0, \frac{1}{2}, 0\right\}$ & $\{0,0,1,0\}$ \\
\hline
\end{tabular}

Table 4: Reduction of terms using Figure 2 for example in Figure 1

The corresponding numbers for AvgDist distance were 23 and 6 respectively. The MinDist returned 505 object pairs with distance 0 as many objects shared one or more terms. Consequently, the top- $k$ lists returned were arbitrary. This convinced us of the quality of the EMD and its usefulness in finding the top- $k$ similar pairs of objects described by terms on tree ontologies. Nevertheless, the two other distance measures have been proved to be useful in specific contexts [2, 14].

We next design algorithms to efficiently compute the top- $k$ pairs using these distances. We start with the EMD as it is the most interesting and useful measure.

\section{The Algorithm for EMD}

When the two sets contain $N$ objects each, the problem of finding top- $k$ pairs of objects can be solved by performing $O\left(N^{2}\right)$ EMD computations. However, the prohibitive time required by each EMD computation makes the entire running time $\left(O\left(N^{2}\right) \times O(E M D)+O\left(N^{2} \log N\right)\right)$ impractical 3

\subsection{Lower Bound using Reduced Number of Terms}

When the ontology tree has a size of $T$, the ground distance matrix is of size $T^{2}$. However, we need not consider all the terms as we can prune the terms that are absent in either of the object descriptions 4 Thus, the number of flow variables is quadratic in the size of the object descriptions. This is still impractical: the average time taken to compute $d_{e m d}$ for objects of size 7 was found to be $54 \mathrm{~ms} 5$

Since the complexity of EMD depends mainly on the number of flow variables, which is quadratic in the number of terms by which each object is described, the run-

\footnotetext{
${ }^{3}$ The sorting of $N^{2}$ pairs require an additional $O\left(N^{2} \log N\right)$ time.

${ }^{4}$ The row and column sums for these terms in the flow matrix will be 0 and hence all the flows will be 0 individually as well.

${ }^{5}$ All the times reported in the paper are based on a $3 \mathrm{GHz}$ machine with 2 GB of RAM running Fedora Linux 9.
} 


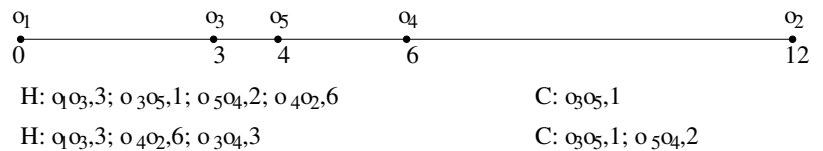

Figure 3: Example of ordered generation of object pairs for one dimension.

ning time can be reduced if the size of the object descriptions is reduced. Figure 2 shows how such reduction can be accomplished. The ontology tree is pruned at height 1 ; only the root term and its immediate children remain. When a term thus deleted appears in an object description, it is replaced by its ancestor that is retained. Hence, all the terms in the dashed subtrees in the figure are removed and replaced by the root of the subtrees. The size of an object description is now upper bounded by the branching factor of the root. Table 4 shows the reduced object descriptions.

The EMD between two objects calculated using the reduced ontology is a lower bound of the EMD using the full ontology [12]6. The number of terms in the reduced ontology is generally much less (say $t \ll T$ ), thus reducing the number of flow variables to $t^{2}$. Since the complexity of linear programming is at least super-linear in the number of flow variables, the running time of EMD decreases by a large factor of $T^{2} / t^{2}$. The number of distance computations, however, still remains $O\left(N^{2}\right)$. Next, we show how to reduce the number of distance computations.

\section{2 $\mathrm{L}_{1}$ Lower Bound}

The $L_{1}$ distance, when scaled by the sum of the total mass, can be used as a lower bound for EMD [1]. Hence, the $L_{1}$ distance between two objects computed using all $T$ terms, when divided by 2 , serves as a lower bound for EMD between the objects. From now on, whenever we mention $L_{1}$, we mean the scaled version of it which is a lower bound. $L_{1}$ on all terms, in turn, is lower bounded by $L_{1}$ on reduced number of terms. The proof uses the fact that $\left|a_{i}-b_{i}\right|+\left|a_{j}-b_{j}\right| \geq\left|\left(a_{i}+a_{j}\right)-\left(b_{i}+b_{j}\right)\right|$, i.e., when the values are combined, the difference of the sums is more than the sum of the differences. Therefore, $L_{1_{t}}\left(O_{i}, O_{j}\right) \leq L_{1_{T}}\left(O_{i}, O_{j}\right) \leq E M D_{T}\left(O_{i}, O_{j}\right)$, where the subscripts denote the number of terms used. Since $L_{1}$ is much faster to compute (for 3 terms, it takes only $0.002 \mathrm{~ms}$ ), we can calculate a lower bound on EMD for each object pair and then use it as a filtering step to prune many of the pairs.

\footnotetext{
${ }^{6} \mathrm{~A}$ lower bound can be obtained by pruning the tree at any height However, there is a trade-off between the tightness and computational efficiency of the lower bound.
}

The $L_{1}$ distance between two objects is a sum of the distances between the corresponding values in each dimension; therefore, if the distances for all the object pairs are obtained and sorted for each dimension, the threshold algorithm (TA) [7] can be applied to obtain the object pairs with the least sum of distances or the least $L_{1}$ distance in a progressive manner. The order of obtaining increasing $L_{1}$ distances can then be used as a guide to order the EMD computations of the object pairs.

Obtaining a sorted list of object pairs for each dimension requires $O\left(N^{2} \log N\right)$ time. TA, however, also works when the next object pair in the list can be output in a sorted manner whenever needed. This avoids the $O\left(N^{2}\right)$ computations. Hence, now our problem is reduced to outputting the next smallest pairwise distance whenever asked for in a particular list (or dimension).

For this, we maintain two data structures for each dimension: (i) a min-heap $H$ that outputs the next best pair, and (ii) a list $C$ that stores all the pairs that have been outputted from $H$.

Initially, the $N$ objects are sorted and all $N-1$ consecutive object pairs (not necessarily $O_{i} O_{i+1}$ ) corresponding to $N-1$ differences are inserted into $H$. Figure 3 shows an example. The 5 objects are sorted according to their values for the dimension that is being processed. Initially, $H$ contains the 4 object pairs corresponding to the 4 differences in the sorted list. Whenever the next pair is asked for by TA, the minimum object pair from $H$ is extracted and returned. It is also inserted into $C$. In this example, after the first call, $\mathrm{O}_{3} \mathrm{O}_{5}$ is extracted from $\mathrm{H}$ and inserted into $C$. Similarly, in the next call, $O_{5} O_{4}$ is extracted.

The initial pairs are not sufficient though. There may be a non-initial pair (e.g., $\mathrm{O}_{3} \mathrm{O}_{4}$ ) with a value (3) less than that of an initial pair $\left(\mathrm{O}_{4} \mathrm{O}_{2}\right.$ with value 6$)$. However, the important point to note is that any non-initial pair is a combination of some of the initial pairs. Two pairs which have an overlapping object can be fused together to generate a new pair. For example, $\mathrm{O}_{3} \mathrm{O}_{4}$ can be generated from $\mathrm{O}_{3} \mathrm{O}_{5}$ and $\mathrm{O}_{5} \mathrm{O}_{4}$ since $\mathrm{O}_{5}$ is overlapping. Further, a pair can never be the least pair until and unless the pairs from which it has been generated have been chosen (i.e., output from $H$ ). Therefore, in the example, $O_{3} O_{4}$ is added to $\mathrm{H}$ only after both $O_{3} O_{5}$ and $O_{5} O_{4}$ have been chosen. In general, when a pair $O_{x} O_{y}$ is chosen, the contents of $C$ are scanned and new pairs are generated if possible. If $C$ has pairs of the form $O_{w} O_{x}$ and $O_{y} O_{z}$, new pairs $O_{w} O_{y}$ and $O_{x} O_{z}$ are generated respectively and are inserted into $H$. The value of this new pair is the sum of the values of the pairs from which it is generated. 


\subsection{Algorithm}

Figure 4 summarizes the entire EMD algorithm that uses TA with the lower bounding strategy. First, the $L_{1}$ lower bound using reduced number of terms is extracted from the heap (line 14). If it is less than the current $k^{\text {th }}$ estimate P.dist in $P$ (line 15), the bound is improved by computing the $L_{1}$ using all the terms (line 16). If it still less (line 17), the exact EMD is computed (line 18) and the top- $k$ list is modified, if necessary (line 21). For each such $L_{1}$-reduced computation, the threshold distance $(R)$ is increased. When $R>k^{\text {th }}$ distance in $P$, no other object pair can have $L_{1}$-reduced distance less than the top- $k$ pairs already found. Therefore, the EMD distances will also be greater. Hence, the algorithm is then halted.

\subsection{Analysis of Time Complexity}

For each of the $t$ dimensions, we incur the following cost. Initially, sorting takes $O(N \log N)$ time 7 Thereafter, inserting the $N-1$ elements in the heap takes $O(N)$ time. With each call to the heap, an extract operation takes $O(\log N)$. At the $i^{\text {th }}$ iteration, at most $i$ elements are added to the heap again. This takes $O(i \log N)$ time. Thus, if we have $k^{\prime}$ calls (this $k^{\prime}$ is generally larger than $k$ as many object pairs with low lower bound but high EMD are examined), the time per dimension is $O\left(k^{\prime 2} \log N\right)$ which leads to a total time of $\sum_{j=1}^{t} O\left(k_{j}^{\prime 2} \log N\right)$ or $O\left(t k_{\max }^{\prime 2} \log N\right)$ where $k_{\max }$ is the maximum of all $k_{j}^{\prime} \mathrm{s}$.

Space Complexity: Heap $j$ requires $O\left(N+k_{j}^{\prime 2}\right)$ space where $k_{j}^{\prime}$ is the number of calls made on column $j$. Hence, the total space required is $O\left(t\left(N+k_{\max }^{\prime 2}\right)\right)$ where $k_{\max }^{\prime}$ is the maximum of $k_{j}^{\prime}$ 's.

\section{The Algorithm for MinDist}

Unlike the $d_{e m d}$ distance, whenever two terms corresponding to two objects are encountered, the MinDist for the object pair can be estimated. If it is better than the current estimate, it is retained; otherwise, it is never needed again. We next explain the MinDist algorithm that exploits this property.

Any object pair $\left(O_{i}, O_{j}\right)$ having a lesser distance than $\left(O_{g}, O_{h}\right)$ must have a term pair $\left(t_{i} \in O_{i}, t_{j} \in O_{j}\right)$ which has a lesser distance than any term pair $\left(t_{g} \in O_{g}, t_{h} \in\right.$ $\left.O_{h}\right)$. Hence, we only need to identify such term pairs $\left(t_{i}, t_{j}\right)$ that are close and process their inverted lists, i.e., the list of objects.

\footnotetext{
${ }^{7}$ An alternative approach using hashing that may reduce this time is discussed in Appendix.
}

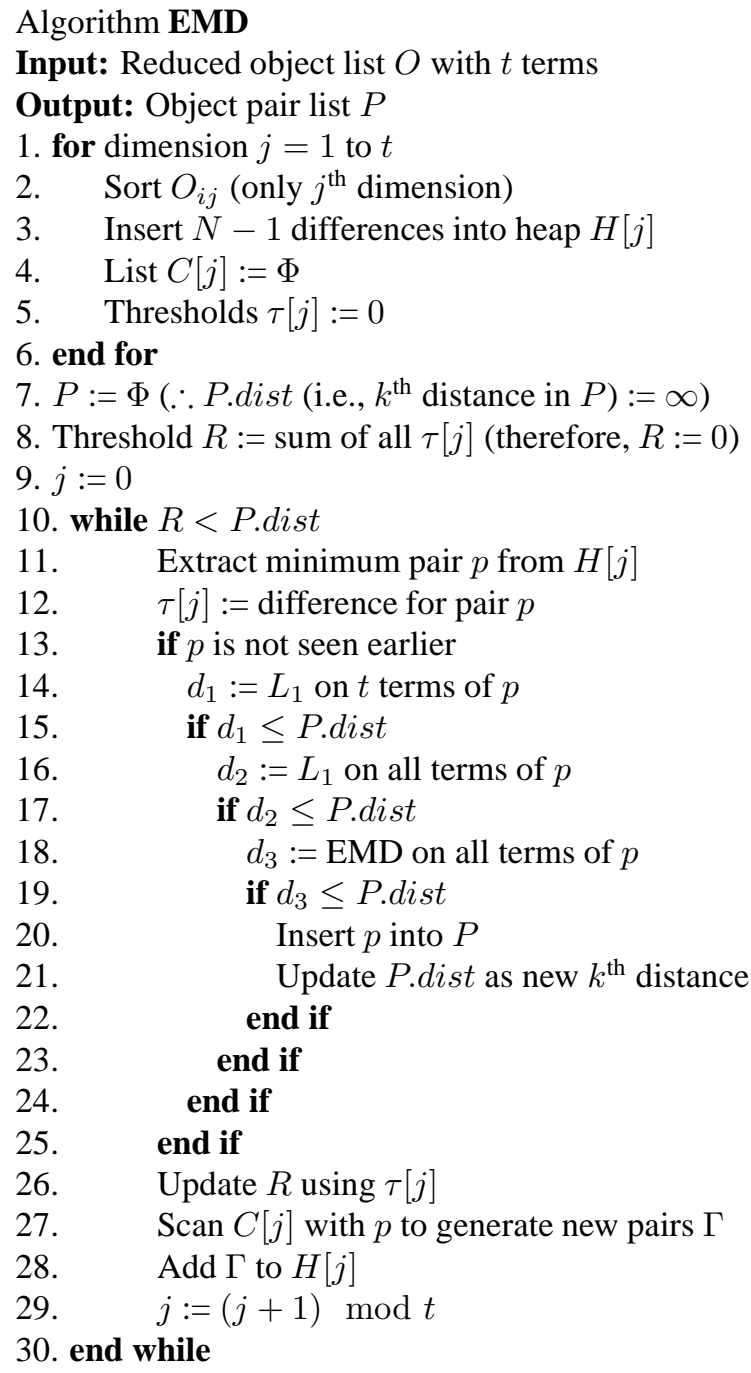

Figure 4: The EMD algorithm.

A pre-processing step is required to build the inverted lists of objects at each node. The inverted index is needed to be built for the minimum and average pairwise distances but not the earth mover's distance. For each object $O_{i}$, when a term $t_{j}$ appears in it, $O_{i}$ is inserted into the inverted list of $t_{j}$. The list is accessed using hashing, and the object is inserted at the top of list.

Figure 5]describes the entire algorithm. For a node, the MinDist algorithm computes the top- $k$ object pairs that are described by at least one term pair in its subtree. Any such object pair must either (i) be in the top- $k$ list of the children, or (ii) contains terms from different subtrees of the children. The recursive definition of the first kind allows us to employ a divide-and-conquer approach. For the 


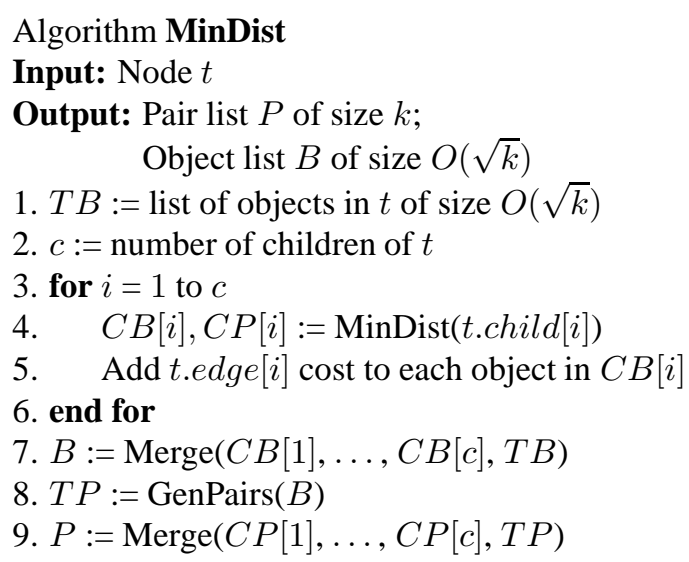

Figure 5: The MinDist algorithm.

second kind, we need a list of objects that are close to the subtree of the children nodes. The lists can then be joined to generate the necessary object pairs. Thus, the MinDist algorithm computed at the root of the ontology returns the top- $k$ pairs.

As shown in Figure 5, each node $t$ maintains two lists: (i) a list of pairs of objects $P$ ordered by their $d_{\min }$ distances; and (ii) a list of objects $B$ ordered by their minimum distances $d_{i}$ to the node $t$. The length of $P$ is at most $k$. The length of $B$ should be enough to ensure that $k$ distinct pairs of objects can be generated from $B$. The number of terms required to do that is $k^{\prime}=O(\lceil\sqrt{k}\rceil)$ 8

When MinDist is called on a node $t$, it selects $k^{\prime}$ objects from its list $L$ into $T B . L$ is the list of objects associated with that term. MinDist is then called on each of its $c$ children. The cost of the edge from $t$ to its child is added to the objects in the corresponding child's object list (line 5). This is done to ensure that the distances are maintained correctly. The $c$ sorted object lists and the list of objects in $t$ are then merged to produce the sorted list $B$.

The merging (line 7) is done using a heap data structure [4]. The heap is initialized with $c+1$ elements at position 1 of each of the child lists and the list $T B$. The minimum element is then extracted into $B$. Since all the individual lists are sorted, the properties of heap guarantee that the object extracted has the least $d_{\text {min }}$ distance from this node. The object at the next position of the list from where this minimum object came is then inserted into the heap. This is repeated $k^{\prime}$ times.

All the possible $k$ pairs are then generated from the $k^{\prime}$ objects in $B$ (method GenPairs in line 8). This list $T P$ computes the $k$ best distances of the object pairs which

\footnotetext{
${ }^{8}$ Since $k^{\prime}\left(k^{\prime}-1\right) / 2 \geq k$, the actual number of terms required is $k^{\prime}=\lceil 1 / 2+\sqrt{1 / 4+2 k}\rceil$.
}

are not in any of the subtrees.

$T P$ is finally merged with the pair lists $C P[i], i=$ $1 \ldots c$ from the children to produce the final pair list $P$ using a heap in the same manner as above (line 9).

\subsection{Analysis of Time Complexity}

In this section, we analyze the time and space complexities of the MinDist algorithm.

We first analyze the time required to compute the inverted index. The object descriptions are read once, and for each term in an object, the corresponding list is accessed in $O(1)$ time using hashing, and the object is inserted at the top of list in another $O(1)$ time. The total time required for this phase is, therefore, $O(D)$.

We next analyze the running time for the main phase of the algorithm. Selecting $k^{\prime}$ objects in $T B$ requires $O\left(k^{\prime}\right)$ time. Adding the child edge costs to each object in $C B$ lists takes $O\left(k^{\prime} c\right)$ time.

At every step of the merging operation, the object with the minimum distance is extracted from the heap and another object is inserted. The size of the heap is, therefore, never more than $O(c)$. Extracting the minimum element and inserting another object into the heap takes $O(\log c)$ time. Since the operation is repeated $k^{\prime}$ times, the total running time of the merging procedure is $O\left(k^{\prime} \log c\right)$.

If, however, the objects in the child lists are not unique, $k^{\prime}$ operations may not be enough to select $k^{\prime}$ different objects. Thus, a hashtable is used to ensure that an object is inserted into the heap only once. First, all the lists are scanned in $O\left(k^{\prime} c\right)$ time. If an object appears for the first time, it is inserted into the hashtable with the object identifier as the key and the distance as the value. If an object appears twice, the one with the minimum distance is maintained in the hashtable. Before any object is inserted into the heap, the hashtable is checked. If this object is different from the one maintained in the hashtable, then there exists another copy of this object with a smaller distance. Hence, this object does not need to be considered. This limits the number of heap operations to $O\left(k^{\prime}\right)$. Assuming that the hashtable operations take constant time, the running time then is $O\left(k^{\prime} \log c\right)$.

Sorting $k$ local object pairs requires $O(k \log k)$ time.

Finally, the sorted pair lists at the node and the children are merged in $O(k c+k \log c)$ time using a heap and a hashtable in a similar manner as before.

Thus, the total running time of the MinDist algorithm at a node with $c$ children is $O(k c+k \log k)$.

The algorithm is run once at each node of the ontology. Assuming that there are $T$ terms in the ontology, the total number of children for all the nodes is $O(T)$. Hence, the 
amortized cost is $O(T k+T k \log k)=O(T k \log k)$.

The total running time of the MinDist algorithm is, therefore, $O(D+T k \log k)$.

Space Complexity: Each node in the ontology contains an object list of size $O\left(k^{\prime}\right)$ and a pair list of size $O(k)$. Once these lists are sent to the parent, they are no longer required. Thus, at any time, the space requirement at a node is $O\left(c\left(k^{\prime}+k\right)\right)$. The total space complexity, therefore, is $O\left(c_{\max }\left(k+k^{\prime}\right)\right)$ where $c_{\max }$ is the largest branching factor of a node in the tree. The inverted index requires $O(D)$ space for storage.

\section{The Algorithm for AvgDist}

Unlike the MinDist algorithm that needs to maintain only one term pair for each object pair, the $d_{\text {avg }}$ distance needs to remember all the possible term-pair distances. Consequently, it runs in two phases: (i) the Build phase, when pertinent information about objects are collected at the root in a bottom-up manner, and (ii) the Query phase, when such information is used to identify the top- $k$ pairs in a top-down order.

For any pair of objects, there are two types of costs that need to be accumulated. The first is the across-tree costs, i.e., the distances between the describing terms that occur in different subtrees of the root, and the second is the within-tree costs, i.e., the distances between the describing terms that are within the same child of the root. For example, in Figure 1, the total pairwise term distances for $\left(\mathrm{O}_{1}, \mathrm{O}_{4}\right)$ can be broken into 2 parts: (i) the across-tree distances between $t_{1}$ of $O_{1}$ and $t_{6}, t_{8}$ of $O_{4}$ in the different subtrees under $t_{1}$ and $t_{2}$ respectively, and (ii) the within-tree distances between $t_{7}$ of $O_{1}$ and $t_{6}, t_{8}$ of $O_{4}$ in the same subtree under $t_{2}$.

To estimate the across-tree distances for object pairs at a node, the following information need to be calculated for each object: (i) the number of terms in the subtree that describe the object, and (ii) the total distance of all such terms to this node. This information is accumulated at the root of the ontology by the build phase AvgDistBuild, which we describe in Section 6.4. After this phase, the root has collected the following tuple for each object: $\left(O_{i}, n_{i}, w_{i}\right)$.

Before describing the two phases of the algorithm, we explain how lower bounds for the across-tree costs of an object pair can be computed using the above information and how such lower bounds can be generated in an ordered manner.

\subsection{Lower Bounds for Across-Tree Costs}

The estimates of the across-tree distances of a pair of objects $O_{i}$ and $O_{j}$ at a node $t$ depend on the occurrences of their describing terms. The span of an object is defined to be the number of subtrees of the root where its constituent terms occur. It can be either single, i.e., its terms occur in only one subtree, or multiple, i.e., its terms occur in multiple subtrees. Based on these, 3 different cases need to be considered. In each case, we would like to write the bounds at a node in terms of the parameters maintained for $O_{i}$ and $O_{j}$ at the node, i.e., in terms of $\left(O_{i}, n_{i}, w_{i}\right)$ and $\left(O_{j}, n_{j}, w_{j}\right)$.

Case 1: Both the objects have single spans. Two subcases need to be considered.

Sub-case 1(a): The objects are in the same subtree. The across-tree cost is 0 and nothing can be concluded about their distance in the subtree without descending deeper into the subtree. Hence, the lower bound is

$$
d_{l b}=0
$$

Sub-case 1(b): The objects are in different subtrees. In this case, the across-tree distance can be estimated exactly. The distance between a term $t_{i} \in O_{i}$ and $t_{j} \in O_{j}$ is $d\left(t_{i}, t\right)+d\left(t_{j}, t\right)$ where $t$ is the node at which this lower bound is being computed. The total across-tree distance is obtained by adding all such combinations of terms:

$$
\begin{aligned}
& \sum_{j=1}^{\left|O_{j}\right|} \sum_{i=1}^{\left|O_{i}\right|} d\left(t_{i}, t\right)+\sum_{i=1}^{\left|O_{i}\right|} \sum_{j=1}^{\left|O_{j}\right|} d\left(t_{j}, t\right) \\
= & n_{j} \cdot \sum_{i=1}^{\left|O_{i}\right|} d\left(t_{i}, t\right)+n_{i} \cdot \sum_{i=1}^{\left|O_{i}\right|} d\left(t_{j}, t\right) \\
= & n_{j} \cdot w_{i}+n_{i} \cdot w_{j}
\end{aligned}
$$

Thus, the average distance is

$$
d_{l b}=d=\frac{w_{i}}{n_{i}}+\frac{w_{j}}{n_{j}}
$$

Since the within-tree distance for this pair is 0 , this is the exact distance.

Case 2: Both the objects have multiple spans. The minimum across-tree distance can be estimated in a manner similar to that in Case 1(b). There are at least two pairings of terms of $O_{i}$ and $O_{j}$ that are in different subtrees. Using Eq. (5), the total across-tree costs for these pairings are $w_{i_{1}} . n_{j_{2}}+w_{j_{2}} . n_{i_{1}}$ and $w_{i_{2}} . n_{j_{1}}+w_{j_{1}} . n_{i_{2}}$, where $n_{i_{1}}$, $w_{i_{1}}$ etc. are the number of terms of $O_{i}$ in one subtree and 
its total distance to $t$ from that subtree. The values of $n_{i_{1}}$, $n_{i_{2}}, n_{j_{1}}$, and $n_{j_{2}}$ are at least 1 . Thus, the total across-tree distance is at least $w_{i_{1}}+w_{i_{2}}+w_{j_{1}}+w_{j_{2}}=w_{i}+w_{j}$. The lower bound for the average pairwise distance, then, is

$$
d_{l b}=\frac{w_{i}+w_{j}}{n_{i} \cdot n_{j}}
$$

Case 3: One object $O_{i}$ has a single span, and the other object $O_{j}$ has a multiple span. Similar to Case 2, there is at least one subtree containing terms of $O_{j}$ but not containing terms of $O_{i}$. The total across-tree cost is then the minimum of $w_{i} . n_{j_{1}}+w_{j_{1}} . n_{i}$ and $w_{i} . n_{j_{2}}+w_{j_{2}} . n_{i}$. Similar to Case 2, there is at least one term of $O_{j}$ that is not in the same subtree of $O_{i}$. Thus, $n_{j_{1}}$ and $n_{j_{2}}$ are at least 1. However, without knowing where the terms of $O_{j}$ occur, nothing can be concluded about $w_{j_{1}}$ and $w_{j_{2}}$. Since the terms may occur at the node itself, the estimates for $w_{j_{1}}$ and $w_{j_{2}}$ are 0 . Hence, the total distance is at least $w_{i}$ producing a lower bound of

$$
d_{l b}=\frac{w_{i}}{n_{i} \cdot n_{j}}
$$

\subsection{Generating Ordered Pairs}

Though the above mentioned lower bounds can be computed for a given pair, the cost of computing them for every pair is $O\left(N^{2}\right)$. We would like to avoid such costly online operations. The trick is to separate the parameters of $O_{i}$ and $O_{j}$ in each lower bound such that they can be systematically generated in an ordered manner whenever needed. The order of generation will guarantee that at any point of time, the lower bounds of the pairs not examined will be greater than or equal to the lower bounds of the pairs already generated. In this section, we will discuss ways to achieve this for each of the cases mentioned above.

To identify pairs of objects in the same subtree (Case $1(\mathrm{a})), c+1$ different lists are maintained at the root corresponding to itself and its $c$ children.

To handle Case 1(b), each of these $c+1$ lists of objects are sorted by the average distance $w_{i} / n_{i}$. Given two such sorted child lists, it is guaranteed that the lower bound (which is the sum of the distances) for an object pair at positions $p_{i}$ in the first list and $p_{j}$ in the second list is lower than the estimate of every pair whose positions are $>p_{i}$ and $>p_{j}$. Thus, every time a pair at positions $\left(p_{i}, p_{j}\right)$ is inspected, only its immediate successors $\left(p_{i}+1, p_{j}\right)$ and $\left(p_{i}, p_{j}+1\right)$ need to be considered. Since there are $c+1$ child lists, the number of possible ways of pairing is $c(c+1) / 2$.
The lower bound for Case 2 is not easily separable in terms of parameters of $O_{i}$ and $O_{j}$. It is, however, separable if for an object pair, the number of terms for the objects (i.e., $n_{i}, n_{j}$ ) are known a priori. To do that, the list of objects with multiple spans is partitioned such that each partition contains objects with a particular $n_{i}$. Pairing $O_{i}$ and $O_{j}$ and knowing which partitions they come from immediately defines the denominator of the lower bound. Thus, if there are $r$ partitions, sorting each partition by $w_{i}$ and performing $r^{2}$ pairings in the same way as done for Case 1(b) orders the pairs according to their lower bounds.

Case 3 is handled similarly. The single-span list is broken into $c+1$ lists and the multiple-span list into $r$ partitions. Generating all $r(c+1)$ pairings gives the lower bounds in an ordered manner.

We next describe how the Query phase of the AvgDist algorithm uses these lower bounds.

\subsection{Query Phase}

The AvgDist-Query procedure (Figure 6) is run at the root of the ontology. It outputs a list $P$ of top- $k$ object pairs. When the size of $P$ is less than $k, P$.dist is $\infty$; otherwise, it is maintained as the $k^{\text {th }}$ largest distance in $P$.

The list $L$ of objects is broken into $c+1+r$ lists corresponding to single and multiple spans as explained in the earlier section. From these lists, the initial pairs with the lower bounds are generated (method GenInitialPairs in line 6) and put into a heap $H$. See Section 6.2 for details on how to generate these pairs.

The top-down searching for object pairs proceeds in a manner where at every stage, only the current "best" pair is examined [10]. Thus, this search strategy is called the best-first search.

The algorithm progresses by extracting the current best pair from the heap, i.e., the pair $p$ with the current best lower bound (line 8). If the lower bound is an estimate for $p$ and not an exact distance as in Case 1(b), the bound can be improved in two ways (line 11). First, the withintree costs at the subtrees in the next level can be estimated again using Eqs. (4) by descending into the subtree (denoted as AvgDist-NextEstimate). The descent is made in a breadth-first order on the tree 9 The second way is to compute the term-wise distances fully without resorting to recursion (denoted as AvgDist-Complete). This, however, disregards the structure of the ontology.

If the exact distance of $p$ is computed, the list $P$ is examined. If the $k^{\text {th }}$ distance in $P$ is more than that of $p$ (line

\footnotetext{
${ }^{9}$ Any order, e.g., depth-first order, will also work. However, if the edge distances decrease exponentially, breadth-first ordering produces better bounds.
} 


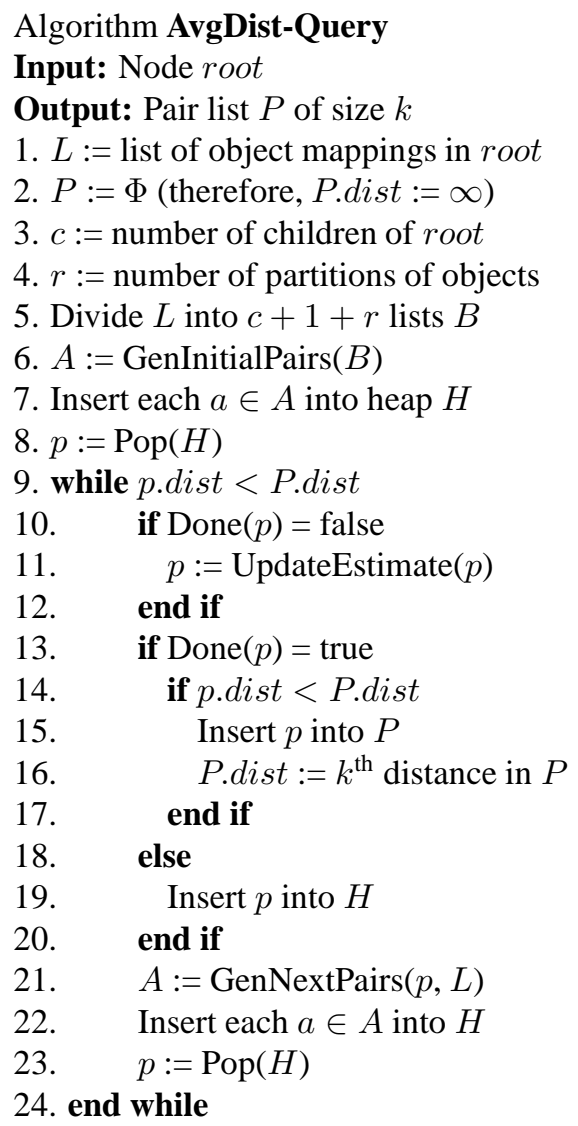

Figure 6: The Query phase of the AvgDist algorithm.

14), $p$ is inserted into $P$ and $P$.dist is modified. The size of $P$ is maintained to be at most $k$ by removing the pair with the largest distance.

If, however, the lower bound of $p$ is still an estimate, $p$ is re-inserted back into the heap $H$ (line 19). The next pairs are generated from the $c+1+r$ lists (method GenNextPairs in line 21 as described in Section 6.2 and in serted into the heap (line 22).

In the next iteration, the pair which is now the best is examined (line 23). If this pair has a distance more than the $k^{\text {th }}$ distance in $P$ (i.e., P.dist), it is guaranteed that all the pairs currently in the heap and all the pairs that are not generated will have a greater distance. This is due to the properties of the heap and the ordered nature of generating the pairs from the $c+1+r$ lists. Thus, the algorithm is then terminated correctly.

\begin{tabular}{|c||c|c|}
\hline Name & $\begin{array}{c}\text { Number of } \\
\text { GO Terms }(T)\end{array}$ & $\begin{array}{c}\text { Number of } \\
\text { Genes }(N)\end{array}$ \\
\hline Process & 13762 & 3437 \\
Function & 7803 & 1958 \\
Localization & 1990 & 645 \\
\hline
\end{tabular}

Table 5: The Gene Ontology (GO) datasets.

\subsection{Build Phase}

In this section, we describe how AvgDist-Build computes the information $\left(O_{i}, n_{i}, w_{i}\right)$ for an object 10 Each node $t$ maintains an inverted list $L$ of objects $O_{i}$ described using $t$. First, it converts $L$ into $B$ by making $n_{i}=1$ and $w_{i}=0$ for each $O_{i} \in L$. Then, it calls AvgDist-Build for each of its children. For each list $C B$ that it receives from a child, and for each object $O_{j} \in C B$, it modifies $w_{j}$ by adding to it the distance to the child node multiplied by the number of times $O_{j}$ occurs in the child subtree, i.e., $w_{j}=w_{j}+$ dist $\times n_{j}$, where dist is the edge distance from $t$ to its child. This ensures that the total distance from $t$ is maintained correctly, since each of the $n_{j}$ objects have to traverse the distance dist.

Analysis of Space and Time Complexities: Assume the total size of the object description to be $D$ which is at most $N \times T$ where $N$ is the total number of objects, and $T$ the total number of terms. The inverted index requires $O(D)$ time and space to construct. We next analyze the space and time complexity of AvgDist-Build in terms of these parameters.

Each object's information is stored at the terms describing it. The information stored in a term is repeated along all its ancestors. Since the size of the description is $D$, and there are $O(\log T)$ ancestors (assuming the ontology to be balanced), the storage cost is $O(D \log T)$.

The running time can be analyzed similarly. At the leaf level of the tree, there are $D$ describing terms. When this $O(D)$ information is sent up to the next level, the time required to combine the information is still $O(D)$ since each object description is read only once and is matched using a hashtable to the information already computed. Assuming the height of the tree to be $O(\log T)$, the total running time is $O(D \log T)$. 


\section{Experiments}

\subsection{Datasets}

We have experimented with real as well as synthetic datasets. The real dataset is that of Gene Ontology (GO, http://www.geneontology.org/). There are three ontologies in GO, corresponding to biological process, molecular function and cellular component (localization) of terms. The details of the three ontologies are given in $\mathrm{Ta}-$ ble 5. The datasets were curated by hashing gene descriptions using their bit-vector representations of the terms and removing the identical genes.

The synthetic datasets were generated by controlling the number of objects, the number of terms, the average branching factor of the ontology tree and the average number of terms per object. The ontologies and the object datasets are created separately. Ontologies have a fixed size and an average branching factor. Starting from the root, we generate a random number of children by perturbing the average branching factor within some limits. We continue with this at all successive nodes. The object dataset is generated with a fixed number of objects and an average number of terms per object. Again, a random number is generated from the average by perturbing it. Then, terms are picked from the ontology randomly without replacement for the required number of terms. This process is repeated for all objects.

\subsection{Experimental Setup}

When the distance function between the objects is defined as the earth mover's distance the following schemes were evaluated:

- $L_{1}$-reduced: In this scheme (Section 4), the $L_{1}$ on reduced number of terms is used.

- $L_{1}$-full: In this scheme, the $L_{1}$ on all terms is used. The tree is not pruned at a height 1 .

- EMD-reduced: All the $O\left(N^{2}\right)$ EMDs on reduced number of terms are computed. These are then used to prune those object pairs for which the reduced EMD is greater than the $k^{\text {th }}$ best EMD already found.

- Brute-force: In this scheme, all the $O\left(N^{2}\right)$ pairs are computed and then the top- $k$ pairs are returned.

The performance of the brute-force scheme $(267 \mathrm{~s}$ for $N=100$ objects) is too impractical to be of any use and are, therefore, not reported. Also, the times of $L_{1}$-full are

\footnotetext{
${ }^{10}$ Figure 17 in Appendix outlines the algorithm.
}

not reported since, in the best case, it can only save $L_{1}$ reduced computations, which are very fast anyway. In all the experiments, it was actually worse than $L_{1}$-reduced.

When the distance function between the objects is defined as the minimum pairwise distance between the terms, the following schemes were considered:

- MinDist: This is the scheme described in Section 5 that has a running time of $O(T k \log k)$.

- Brute-force: In this scheme, all the $O\left(N^{2}\right)$ pairs are computed and then the top- $k$ pairs are returned. Maintaining a heap of size at most $k$ gives the running time of this scheme to be $O\left(N^{2} \log k\right)$. Due to the exorbitant online costs of it, this scheme is not practically useful.

For $N=10^{4}$, the top- $k$ computation using the brute-force algorithm finishes in $\sim 300 \mathrm{~s}$. Since the MinDist has a better running time, we report the experiments for MinDist only.

When the distance function between the objects is defined as the average pairwise distance between the terms, the following schemes were evaluated:

- AvgDist-NextEstimate: In this variant of AvgDist, the estimate for the best-pair is improved by progressively descending into the subtrees and estimating the across-tree costs at the roots of those subtrees.

- AvgDist-Complete: This is the other variant of AvgDist where the exact distance is computed at one go by computing all the pairwise term distances.

- Brute-force: In this scheme, all the $O\left(N^{2}\right)$ pairs are computed and then the top- $k$ pairs are returned.

The performance of the brute-force scheme ( $300 \mathrm{~s}$ for $10^{4}$ dataset) is much higher than that for AvgDist schemes. Consequently, it is not discussed any further.

Sections 7.3 to 7.7 report experiments on EMD while Sections 7.8 to 7.10 and 7.11 to 7.14 report on MinDist and AvgDist respectively.

\subsection{Effect of $k$ on EMD}

Figure 7 shows the effect of $k$ on the running time of GO localization dataset. When $k$ is increased, more number of $L_{1}$ computations are needed before the TA can halt. Consequently, more number of EMD calculations are also required. 


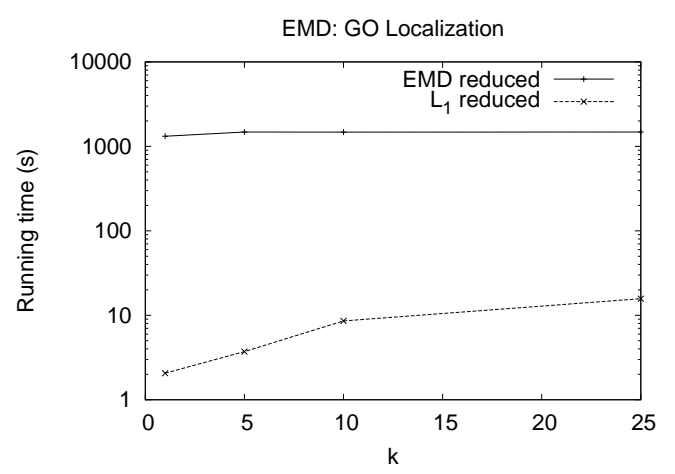

Figure 7: Effect of $k$ on EMD.

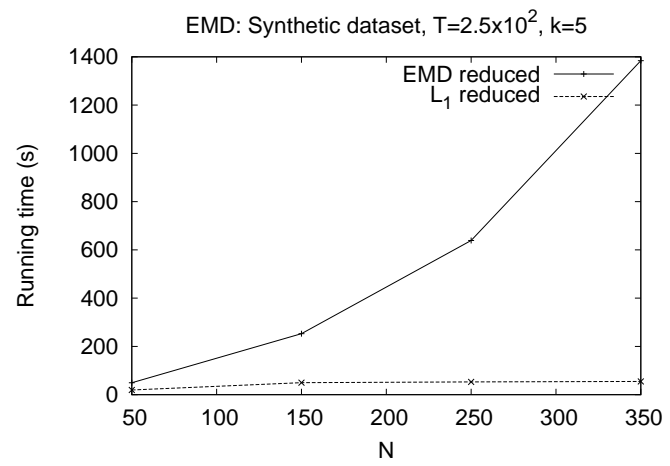

Figure 8: Effect of $N$ on EMD.

\subsection{Effect of N on EMD}

Figure 8 shows that the scalability of our algorithm with $N$ is better than quadratic. Even though the number of objects increases quadratically, due to $L_{1}$ lower bounding, many of the object pairs are pruned. Consequently, the number of full EMD computations increases by a lower factor. Also, even for $N=350$ which translates to $6 \times 10^{4}$ object pairs, our algorithm finishes in only $55 \mathrm{~s}$.

\subsection{Number of Object Pairs for EMD}

To check the effect of increasing $N$, we measured the ratio of object pairs for which full EMD computation was done. The ratio was measured as number of pairs investigated to the total number of possible pairs $(N(N-1) / 2)$ and is denoted by $\eta$. As Figure 9 shows, $\eta$ decreases when $N$ is increased. For $N=250$, the number of EMD computations becomes lower than $10 \%$.

\subsection{Effect of T on EMD}

The next experiment measures the effect of the total number of terms on the EMD computations. Since both the

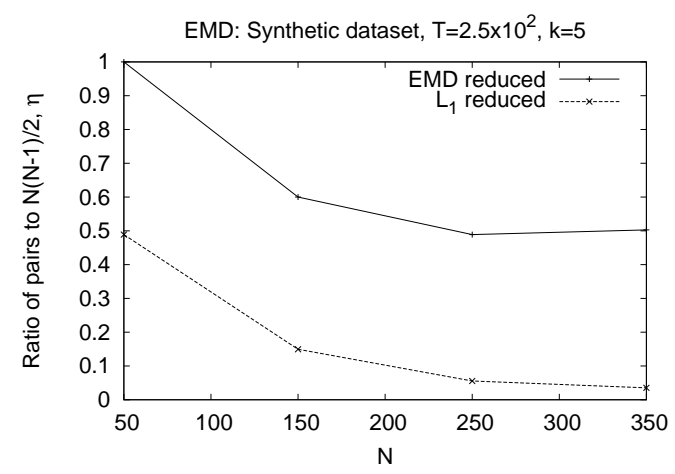

Figure 9: Effect of $N$ on number of pairs examined for EMD.

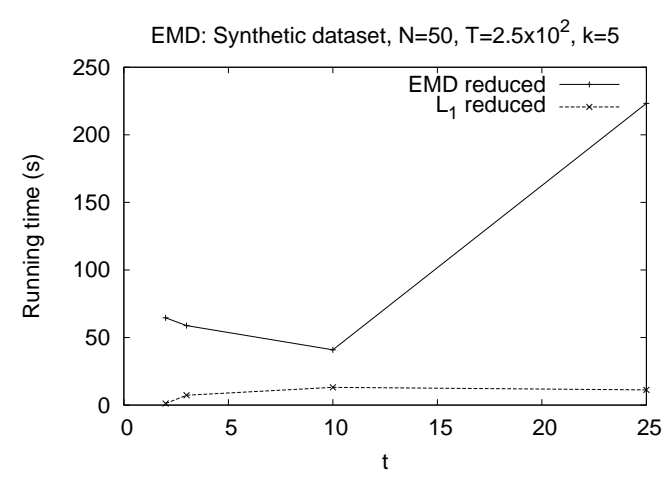

Figure 10: Effect of $t$ on EMD.

$L_{1}$ and EMD-reduced depends only on the reduced number of terms, the effect of $T$ is minimal (graph not shown).

\subsection{Effect of $t$ on EMD}

As the number of children of root, i.e., $t$ increases, the complexity of the TA increases linearly. Figure 10 shows the running times for varying $t$. The size of each object description is limited to 10 . When $t \leq 10$, the time increases. The EMD-reduced behaves in the opposite manner. This is due to the interaction of two opposing effects: as $t$ increases, each computation takes more time, but the lower bound gets tighter as more number of terms are taken into account resulting in less number of full EMD computations. However, when $t>10$, since there are at most 10 terms in each object, the object description size do not get reduced and each EMD-reduced computation takes as much time as the full EMD computation. Since $O\left(N^{2}\right)$ of these computations are performed, the running time shoots up. The $L_{1}$-reduced, on the other hand, shows only a little increase.

The next set of experiments measure the effect of different parameters on the MinDist algorithm. 


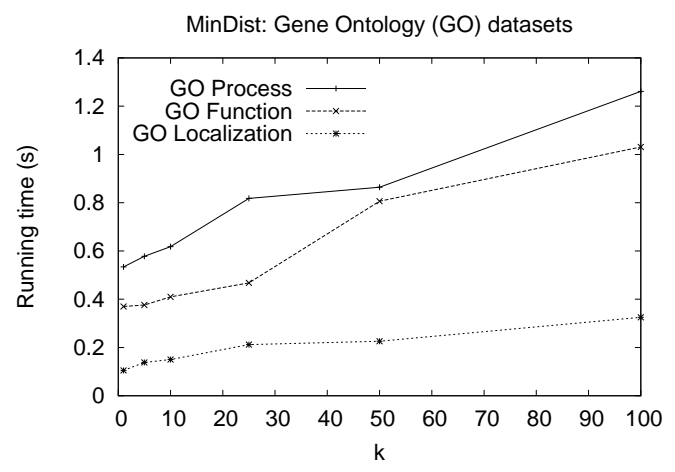

Figure 11: Effect of $k$ on MinDist.

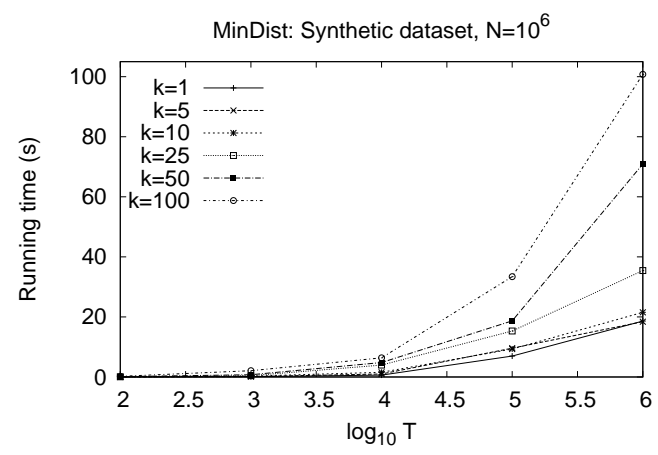

Figure 12: Effect of $T$ on MinDist.

\subsection{Effect of $k$ on MinDist}

The first set of experiments measure the effect of the number of top pairs queried $(k)$, on the running time of the MinDist algorithm. As shown in Figure 11, the scalability of MinDist with $k$ is linear. The analysis done in Section 5.1 shows that for small values of $k$, this is the expected behavior. The largest real dataset-GO processfinishes in less than $1 \mathrm{~s}$ for $k \leq 50$, demonstrating the effectiveness of the algorithm.

\subsection{Effect of T on MinDist}

We next report the effect of the number of terms $T$ on the running time. Figure 12 shows that increasing $T$ increments the running time of MinDist linearly, independent of the value of $k$. We also note the practicality of the MinDist algorithm. For a very large dataset of size $N=10^{6}$ and a very large tree of size $T=10^{6}$, a top100 query finishes in about $100 \mathrm{~s}$. For smaller $k$ 's and for smaller T's, the running time is in seconds.

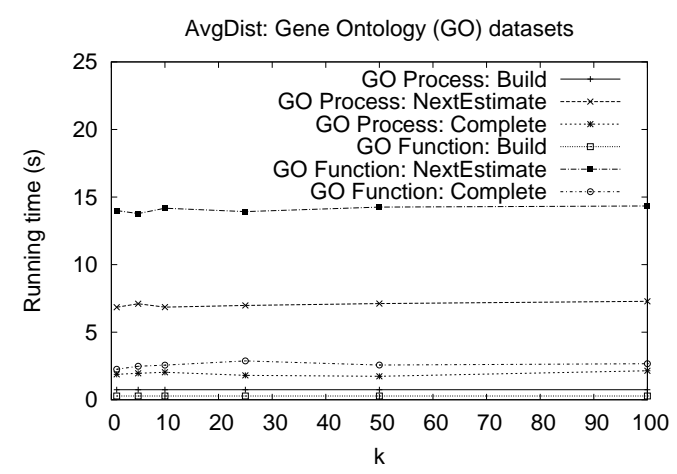

Figure 13: Effect of $k$ on AvgDist.

\subsection{Effect of $\mathrm{N}$ on MinDist}

The running time analysis of the MinDist algorithm shows that it is independent of the number of objects $N$. When the number of terms $T$ is kept constant, the experiments confirm that the running time is practically constant even when $N$ is increased from $10^{3}$ to $10^{6}$ (graph not shown).

The next set of experiments evaluate the performance of the two variants of AvgDist.

\subsection{Effect of $k$ on AvgDist}

The first experiment on AvgDist illustrates the effect of $k$ on the running time of the Build phase and the two different variants-NextEstimate and Complete-for the two larger GO datasets. All the six curves in Figure 13 are relatively flat, showing that the effect of $k$ is minimal. Intuitively, the running time of AvgDist depends on the actual number of object pairs investigated. For the GO datasets, even for even large $k$ 's up to 100 , this remains almost constant. Moreover, the Build phase takes negligible time in comparison to the Query phase.

\subsection{Number of Object Pairs for AvgDist}

We further investigated the effect of $k$ by measuring the number of object pairs that are examined in the Query phase of the AvgDist algorithm. For this, we increased $k$ up to 10000 . Figure 14 shows that $\eta$ (i.e., the ratio to the total number of possible pairs) increases very slowly with $k$. The results are robust across different values of $T$ (as shown in the figure) and $N$ (not shown). This is the reason why the running time is also constant across $k$.

The NextEstimate method examines less than $2 \%$ of the total number of pairs. The Complete method investigates more object pairs (about 7\%) than the NextEstimate method. Computing a distance for the current bestpair guarantees that only those pairs which have a bound 


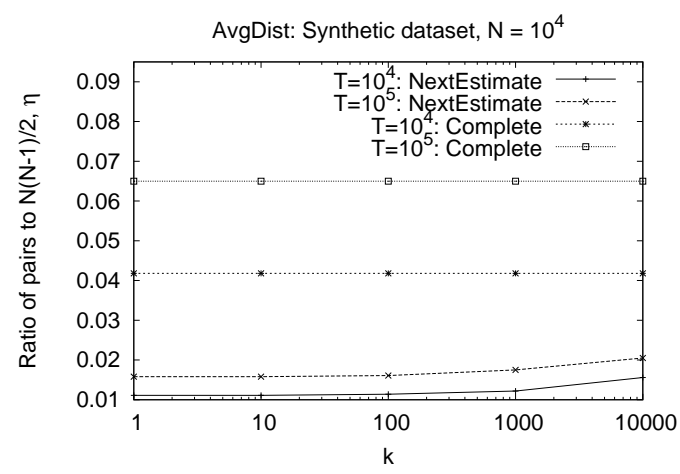

Figure 14: Effect of $k$ on number of pairs examined for AvgDist.

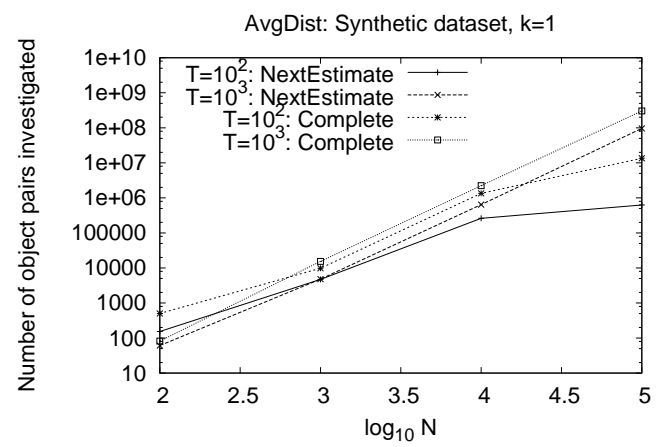

Figure 15: Effect of $N$ on number of pairs examined for AvgDist.

lower than this distance will be analyzed. For the NextEstimate method, the distance of the best-pair is computed progressively, thereby saving on full AvgDist computations as compared to the Complete method, which finds the actual distance of the best-pair.

\subsection{Effect of $\mathrm{N}$ on AvgDist}

We next discuss the experimental results when the number of objects is varied. We first measure the effect of number of objects on the Build phase. From the analysis done in Section 6.4 we expect the running time to grow linearly with the size of the input information. Assuming that the number of describing terms for an object is constant, the size of the information is directly proportional to the number of objects. The experiment shows that the scalability is indeed linear (graph not shown).

The next experiment (Figure 15) shows that the number of pairs investigated grows at most quadratically with $N$. Since the objects are generated using the same random process, this is expected.

\subsection{Effect of T on AvgDist}

The next set of experiments measure the effect of the number of terms $T$ on the different components of the AvgDist algorithm. Figure 16]shows the time taken to complete the Build phase. Note that this phase takes the same amount of time regardless of the choice of the method for estimating the distance of a pair. Since the build procedure is run at each node, the effect of $T$ is linear. Further, as can be seen from the plot, when the number of objects increase, more information needs to be processed at each node and the running time increases linearly.

The next experiment measures the number of pairs investigated against different values of $T$. As shown in Section 7.13, the number of pairs depends primarily on the distribution of the objects on the tree-mainly the number of objects falling in the single span lists-and not on the size of the tree. Consequently, the size of the tree $T$ has no appreciable effect. Similar to the previous set of experiments, this effect of $T$ (or rather the lack of it) is directly reflected in the running time as well. The running time is essentially independent of $T$ (graph not shown).

\section{Conclusions}

In this paper, we proposed the problem of finding top- $k$ most similar object pairs annotated with terms from an ontology. The terms represent concepts and the objects are described using these concepts. The join problem exposed the computational aspects of the domain well.

We then defined and motivated three object distances that can be used to define the dissimilarity (or, equivalently similarity) between a pair of objects. The minimum pairwise distance is useful in order to search objects that share a similar term. The average pairwise distance captures the notion of similarity when the object definitions are imprecise or when objects need to be compared on multiple attributes. The third one, earth mover's distance, is particularly useful as it finds the best way of matching terms in one object with those in the other by capturing the term-to-term relationships, and measures the distance corresponding to this best matching.

Finally, we designed algorithms to efficiently solve the problem using all the above distance measures. The algorithm for EMD uses $L_{1}$ distance as a lower bound and even avoids all $L_{1}$ computations by modifying the threshold algorithm. The algorithm that solves the problem for the minimum pairwise distance runs in $O(D+T k \log k)$ time. For the average pairwise distance, we devised a best-first search strategy that avoids all pairs investigation by generating lower bounds in an ordered manner. Ex- 


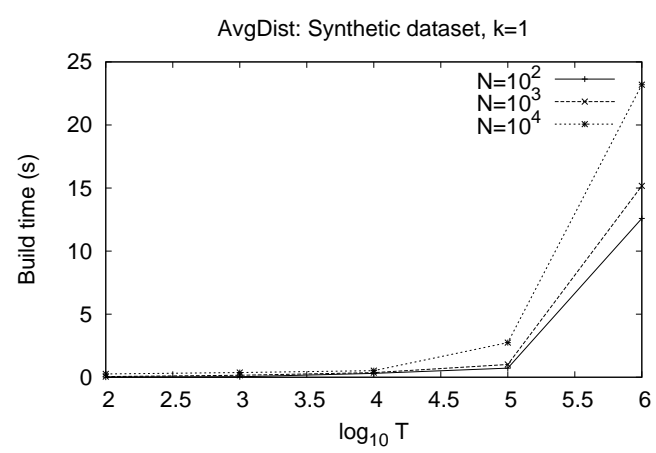

Figure 16: Effect of $T$ on Build phase of AvgDist.

perimental evaluations demonstrated the practicality and scalability of our algorithms.

In future, we would like to design algorithms for other distance measures and lower bounds. We would also like to develop methods that use term statistics to improve the expected running time and further explore the optimal height of pruning the ontology tree for EMD. Lastly, algorithms for $k$-NN and range queries should be simple extensions of the proposed algorithms.

\section{References}

[1] I. Assent, A. Wenning, and T. Seidl. Approximation techniques for indexing the earth mover's distance in multimedia databases. In ICDE, pages 11-22, 2006.

[2] M. Brameier and C. Wiuf. Co-clustering and visualization of gene expression data and gene ontology terms for Saccharomyces cerevisiae using selforganizing maps. J. Biomed. Inf., 40(2):160-173, 2007.

[3] P. Ciaccia, M. Patella, and P. Zezula. M-tree: An efficient access method for similarity search in metric spaces. In $V L D B$, pages 426-435, 1997.

[4] T. H. Cormen, C. E. Leiserson, R. L. Rivest, and C. Stein. Introduction to Algorithms. MIT Press, 2001.

[5] F. M. Couto, M. J. Silva, and P. M. Coutinho. Measuring semantic similarity between gene ontology terms. Data \& Knowledge Engg., 61(1):137-152, 2007.

[6] S. Deerwester, S. T. Dumais, G. W. Furnas, T. K. Landauer, and R. Harshman. Indexing by latent semantic analysis. J. Am. Soc. Inf. Sc., 41(6):391-407, 1990.
[7] R. Fagin, A. Lotem, and M. Naor. Optimal aggregation algorithms for middleware. In $P O D S$, pages 102-113, 2001.

[8] X. Guo, R. Liu, C. D. Shriver, H. Hu, and M. N. Liebman. Assessing semantic similarity measures for the characterization of human regulatory pathways. Bioinf., 22(8):967-973, 2006.

[9] A. Gupta. Embedding tree metrics into low dimensional euclidean spaces. In STOC, pages 694-700, 1999.

[10] G. R. Hjaltason and H. Samet. Distance browsing in spatial databases. ACM Trans. Database Syst., 24(2):265-318, 1999.

[11] E. Johnston and N. Kushmerick. Web service aggregation with string distance ensembles and active probe selection. Information Fusion, 9:481-500, 2008.

[12] V. Ljosa, A. Bhattacharya, and A. K. Singh. Indexing spatially sensitive distance measures using multi-resolution lower bounds. In EDBT, pages 865-883, 2006.

[13] P. W. Lord, R. D. Stevens, A. Brass, and C. A. Goble. Investigating semantic similarity measures across the gene ontology: the relationship between sequence and annotation. Bioinf., 19(10):12751283, 2003.

[14] L. Mariño-Ramírez, O. Bodenreider, N. Kantz, and I. K. Jordan. Co-evolutionary rates of functionally related yeast genes. Evolutionary Bioinf., 2:271276, 2006.

[15] R. Mihalcea and C. Corley. Corpus-based and knowledge-based measures of text semantic similarity. In $A A A I$, pages 775-780, 2006.

[16] J. M. Patel and D. J. DeWitt. Partition based spatialmerge join. In SIGMOD, pages 259-270, 1996.

[17] G. Salton and C. Buckley. Term-weighting approaches in automatic text retrieval. Inf. Proc. \& Mgmt., 24(5):513-523, 1988.

[18] Y. Tao, L. Sam, J. Li, C. Friedman, and Y. A. Lussier. Information theory applied to the sparse gene ontology annotation network to predict novel gene function. Bioinf., 23(13):i529-i538, 2007.

[19] X. Wan and Y. Peng. The earth mover's distance as a semantic measure for document similarity. In CIKM, pages 301-302, 2005. 


\section{Appendix}

\section{A Average pairwise distance follows triangular inequality}

Lemma 1. The average pairwise distance $d_{\text {avg }}$ as defined in Eq. (2) follows the triangular inequality property.

Proof. Assume any three objects $A, B$ and $C$. We need to prove that $d_{\text {avg }}(A, B)+d_{\text {avg }}(B, C) \geq d_{\text {avg }}(C, A)$.

Consider any term $a_{i} \in A, b_{j} \in B$, and $c_{k} \in C$. Since the term distance function is a metric, we can write $d\left(a_{i}, b_{j}\right)+d\left(b_{j}, c_{k}\right) \geq d\left(c_{k}, a_{i}\right)$. Adding the $|A| \cdot|B| .|C|$ equations together yields

$$
\begin{aligned}
\sum_{i, j, k=1}^{|A|,|B|,|C|} d\left(a_{i}, b_{j}\right)+\sum_{i, j, k=1}^{|A|,|B|,|C|} d\left(b_{j}, c_{k}\right) & \\
& \geq \sum_{i, j, k=1}^{|A|,|B|,|C|} d\left(c_{k}, a_{i}\right)
\end{aligned}
$$

or, $|C| \cdot \sum_{i, j=1}^{|A|,|B|} d\left(a_{i}, b_{j}\right)+|A| \cdot \sum_{j, k=1}^{|B|,|C|} d\left(b_{j}, c_{k}\right)$

$$
\geq|B| \cdot \sum_{k, i=1}^{|C|,|A|} d\left(c_{k}, a_{i}\right)
$$

Dividing by $|A| \cdot|B| .|C|$, we get

$$
d_{\text {avg }}(A, B)+d_{\text {avg }}(B, C) \geq d_{\text {avg }}(C, A)
$$

\section{B Hashing}

If $L_{1}$ is computed on all the terms in the TA phase of the EMD algorithm, then the time required for sorting of $N$ objects in the initial phase can be saved. The key is to observe that all values for an object will be of the form $1 / c$ where $c$ is the count of the number of terms in the object. Since $c$ is at most $T$, a hashtable of size $T$ with keys $\frac{1}{1}, \ldots, \frac{1}{T}$ can be maintained. The $N$ object values will be hashed into it. The heap $H$ will be filled up with values of the form $\frac{1}{i}-\frac{1}{i+1}$ only. This requires a running time of $O(N+T)$ instead of $O(N \log N)$.

When reduced number of terms are used, the values will be of the form $\frac{t_{1}}{t_{2}}$, where $1 \leq t_{1} \leq T$ and $1 \leq t_{2} \leq T$. This requires a running time of $O\left(N+T^{2}\right)$.

\section{Algorithm AvgDist-Build}

Figure 17: The Build phase of the AvgDist algorithm.

\author{
Algorithm AvgDist-Build \\ Input: Node $t$ \\ Output: Object list $B$ \\ 1. $L:=$ list of objects in $t$ \\ 2. $B:=\operatorname{Modify}(L)$ \\ 3. $c:=$ number of children of $t$ \\ 4. for $i=1$ to $c$ \\ 5. $C B[i]:=\operatorname{AvgDist-Build}(t . c h i l d[i])$ \\ 6. for each $c o \in C B[i]$ \\ 7. if $\exists o:=\operatorname{Find}(c o . i d, B)$ \\ 8. o.dist $:=$ o.dist + co.dist \\ + co.count $\times$ t.edge $[i]$ \\ 9. o.count $:=$ o.count + co.count \\ 10. end if \\ 11. end for \\ 12. end for
}

